\title{
Locked bilateral posterior fracture-dislocation of the shoulder in an epileptic patient: case report
}

\author{
ENRICO CAUTERO, ENRICO GERVASI
}

Orthopaedics and Traumatology Unit, Latisana Hospital, Latisana (UD), Italy

\begin{abstract}
Bilateral posterior dislocation of the shoulder, often secondary to seizures, is uncommon, while bilateral posterior fracture-dislocations is rarer still: 0.6 cases among a population of 100,000 people per year. The scientific literature contains very few published reports of cases of bilateral posterior fracture-dislocation of the shoulder, a condition that tends to be sustained by epileptic patients during seizures. The authors presented a case of bilateral posterior fracture-dislocation of the shoulder secondary to a first epileptic seizure episode treated by humeral head replacement with stem less implants on both shoulders, with satisfactory radiographic and clinical outcome.
\end{abstract}

Key words: epilepsy, fracture, posterior dislocation, replacement, shoulder.

\section{Introduction}

Epilepsy can be a cause of dislocation and consequent instability of the shoulder. Shoulder dislocations can be locked or recurrent, and muscle contractions or trauma sustained during a seizure episode are the most frequent injury mechanisms (1).

In terms of incidence, posterior dislocation accounts for a small percentage of dislocations occurring in the general population: between 1.7 and $4.3 \%$ (2). Within

\section{Corresponding Author:}

Enrico Cautero, MD

Orthopaedics and Traumatology Unit, Latisana Hospital

Via Sabbionera 45, 33053 Latisana (UD), Italy

E-mail: cauteroenrico@aol.com this group, posterior dislocations associated with fracture are less frequent still: $0.9 \%$ of 1500 shoulder fracture-dislocations according to Neer and Foster (3). Bilateral posterior dislocations, often secondary to seizures, but also reported as a consequence of falls from a height (4-6), are even less common, while bilateral posterior fracture-dislocations are rarer still: 0.6 cases among a population of 100,000 people per year (7). The scientific literature contains very few published reports of cases of bilateral posterior fracture-dislocation, a condition that tends to be sustained by epileptic patients during seizures. Estimates from scientific data suggest that missed or delayed diagnoses of fracture-dislocation of the shoulder are in the order of $60 \%$. Indeed, the diagnosis can be reached only by performing an appropriate trauma-specific series of radiographs (8). Several surgical treatment options have been described: reconstruction of the humeral head with bone grafts $(9,10)$, medial transfer of the subscapularis tendon into the bone defect (4), and anatomical shoulder replacement (11-13).

\section{Case report}

A 46-year-old man presented with locked bilateral posterior fracture-dislocation of the shoulder secondary to a first and only epileptic seizure episode that had lasted many minutes. The dislocation was not diagnosed at the local emergency department (Fig. 1), but eight days later on the basis of the findings of a CT scan (Fig. 2), performed because of persistence of the symptoms. Temporary reduction and pinning was performed. The patient was referred to us because of persistence of the humeral dislocation. Magnetic resonance imaging (MRI) showed, on the right shoulder, an 


\section{oints}

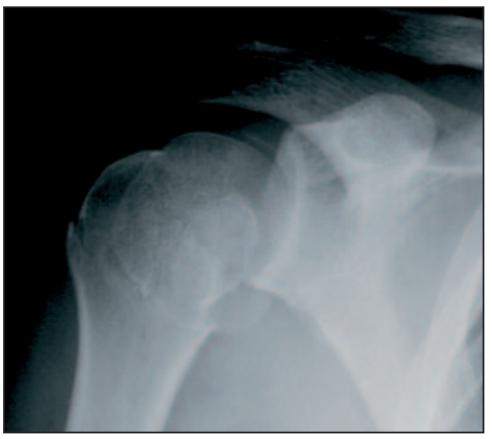

Fig. 1. Right shoulder. The trough line sign, superimposition of the humeral head in the glenoid in the AP view, is a sign of persistent posterior dislocation (by permission of Enrico Gervasi).

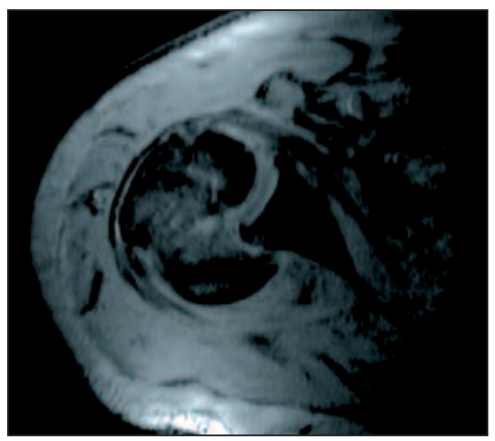

Fig. 3. Axial MR scans of the right shoulder allowed assessment of the defect involving around $40 \%$ of the articular surface of the humeral head (by permission of Enrico Gervasi).

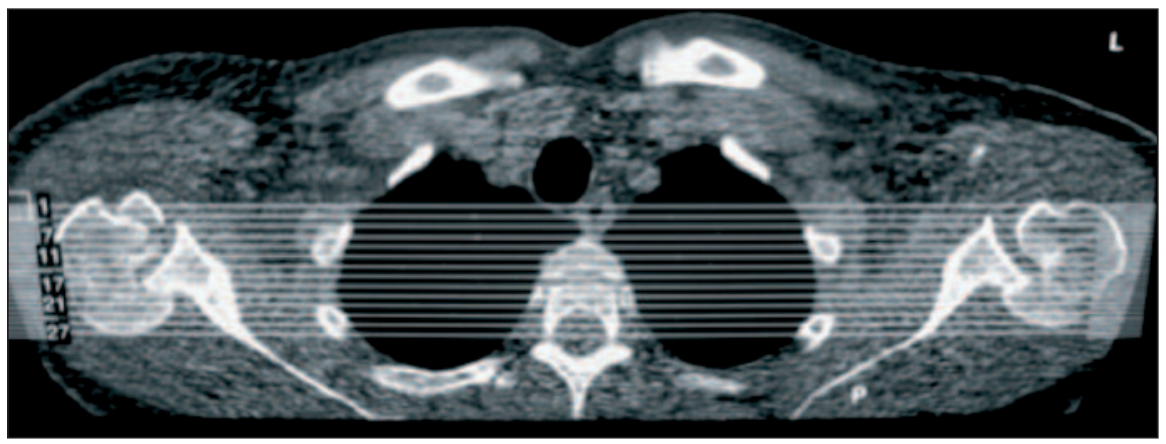

Fig. 2. CT scan showing locked posterior fracture-dislocation of both shoulders with severe damage to the humeral joint (by permission of Enrico Gervasi).
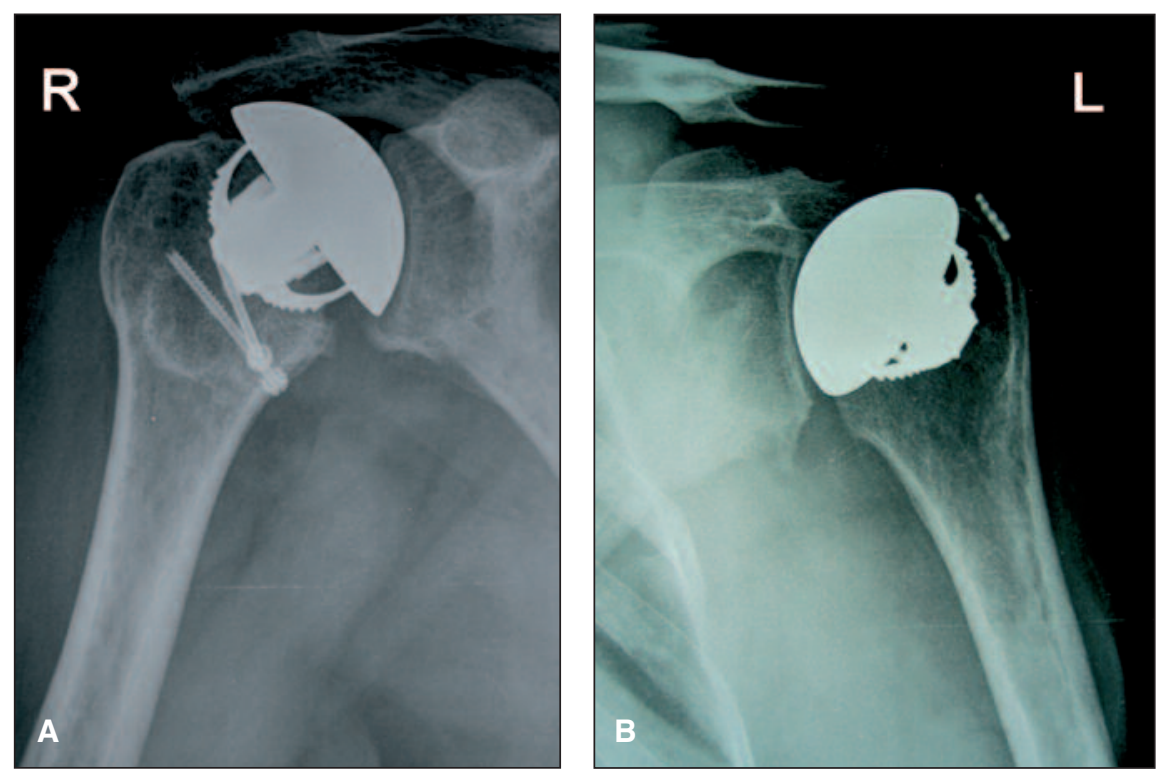

Fig. 4. Radiographic postoperative exams of the right $(A)$ and left $(B)$ shoulder (by permission of Enrico Gervasi) anterior osteochondral defect of the humeral head involving around $40 \%$ of the articular surface (Fig. 3). On surgical exposure, obtained using the deltopectoral approach, the right humeral head confirmed the presence of an anterior osteochondral defect involving around $40 \%$ of the articular surface; it was associated with a traumatic postero-superior rotator cuff tear. Bone grafting of the metaphyseal area was performed using shaped autologous bone harvested from the osteotomized humeral head, and the graft was fixed with two metal screws. Finally, a stemless anatomical prosthesis was implanted. The rotator cuff was repai- red. The left shoulder was operated on six months later using the same type of prosthesis, but without grafting (Fig. 4). Clinical outcome was satisfactory. The patient regained stability and almost complete strength and passive and active range of motion on both shoulders (Fig. 5).

\section{Discussion}

The literature contains very few descriptions of cases of locked bilateral posterior fracture-dislocations of 


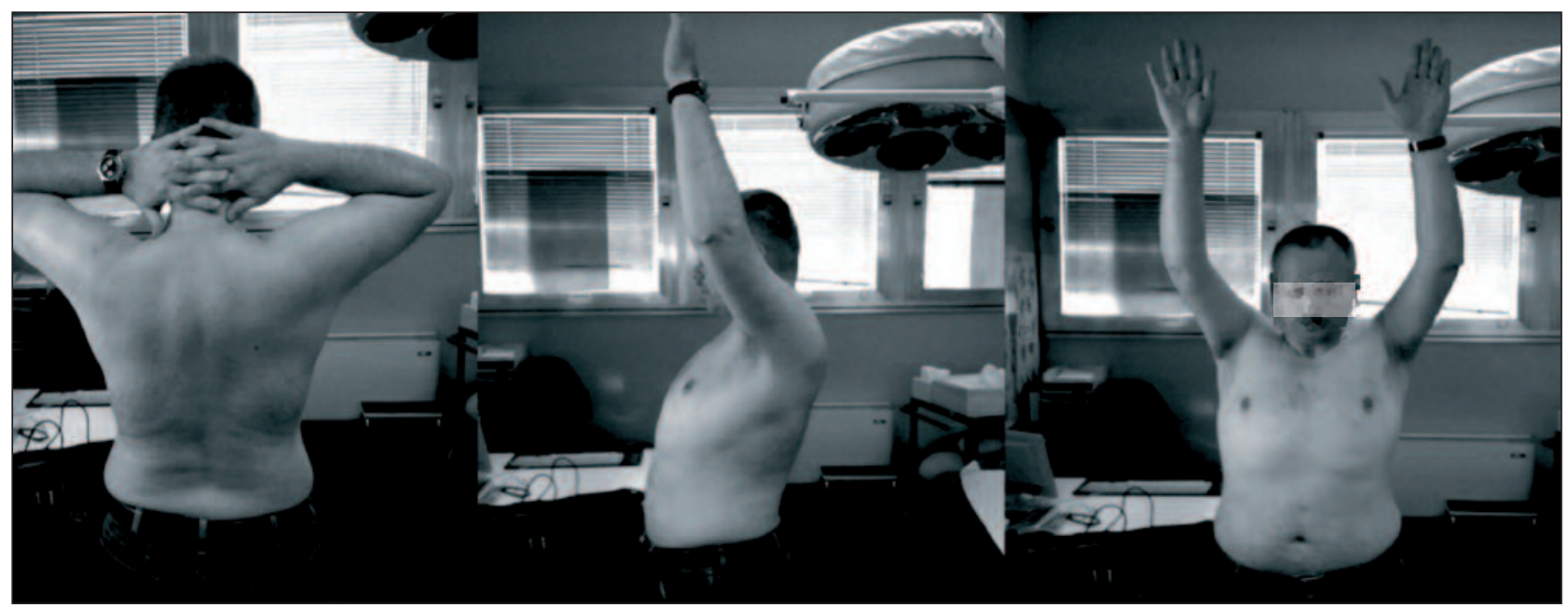

Fig. 5. Satisfactory clinical result at long-term follow-up (by permission of Enrico Gervasi).

the shoulder, a condition usually caused by generalized tonic-clonic seizures in epileptic patients. Other possible causes are road accidents, alcohol-related injuries, and electroshocks administered without curarization. In cases in which the osteochondral injury of the humeral head extends over a volume greater than $20 \%$, surgical treatment is indicated on account of the risk of recurrence of the instability: commonly used methods are anatomical reconstruction of the humeral head with bone grafts, use of cryopreserved allografts, transfer of the subscapularis tendon into the bone defect (according to McLaughlin), postero-inferior capsular shift, and shoulder replacement.

As regards our surgical choice, our decision to use a stem less shoulder prosthesis reflected our desire to spare healthy structures, albeit in the context of prosthetic replacement, which is an invasive procedure.

The autologous bone graft taken from the osteotomized humeral head allowed stable positioning of the prosthesis even in the presence of bone loss (right shoulder).

Despite this patient's good objective and subjective outcome, today we would use two different prostheses: a stem less one on the left, and an anatomical one with a short stem on the right, in order to guard against possible loosening of the implant. This is likely to occur in the event of autologous bone graft resorption, initial signs of which can be seen radiographically.

\section{References}

1. Bühler M, Gerber C. Shoulder instability related to epileptic seizures. J Shoulder Elbow Surg. 2002;11:339-344.

2. Robinson CM, Akhtar A, Mitchell M, et al. Complex posterior fracture-dislocation of the shoulder. Epidemiology, injury patterns, and results of operative treatment. Bone Joint Surg Am. 2007;89:1454-1466.

3. Neer CS 2nd, Foster CR. Inferior capsular shift for involuntary inferior and multidirectional instability of the shoulder. A preliminary report. J Bone Joint Surg Am. 1980; 62:897-908.

4. McLaughlin HL. Posterior dislocation of the shoulder. J Bone Joint Surg Am. 1952;24:584-590.

5. Jacobs BW, Schultz TJ. Shoulder dislocation associated with seizure disorders. J Bone Joint Surg Am. 1970;52: 824.

6. Silbergeld DL, Harkness WF, Bell BA, Bircher MD. Posterior fracture dislocation of the shoulder secondary to epileptic seizures. J R Coll Surg Edinb. 1991;36:139-140.

7. Shaw JL. Bilateral posterior fracture-dislocation of the shoulder and other traumas caused by convulsive seizures. J Bone Joint Surg Am. 1971;53:1437-1440.

8. Devang MG. The trought line sign. Radiology. 2002;224: 485-486.

9. Gerber C, Lambert SM. Allograft reconstruction of segmental defects of the humeral head for the treatment of chronic locked posterior dislocation of the shoulder. J Bone Joint Surg Am. 1996;78:376-382.

10. Chalidis BE, Papadopoulos PP, Dimitriou CG. Reconstruction of a missed posterior locked shoulder fracture-dislocation with bone graft and lesser tuberosity transfer: a case report. J Med Case Rep. 2008;2:260.

11. Sperling JW, Pring M, Antuna SA, et al. Shoulder arthroplasty for locked posterior dislocation of the shoulder. J Shoulder Elbow Surg. 2004;13:522-527.

12. Checchia SL, Santos PD, Miyazaki AN. Surgical treatment of acute and chronic posterior fracture-dislocation of the shoulder. J Shoulder Elbow Surg. 1998;7:53-65.

13. Pritchett JW, Clark JM. Prosthetic replacement for chronic unreduced dislocations of the shoulder. Clin Orthop Relat Res. 1987;216:89-93. 